\title{
VEHICLE DRIVER MODELLING AND SIMULATION BASED ON CONTROLLER APPROACH
}

\author{
Shwetha Baliga \\ Assistant Professor \\ Department of Electronics and Communication Engineering \\ $R V$ College of Engineering \\ Bengaluru, India \\ Pavan Santosh N R \\ UG Student \\ Department of Electronics and Communication Engineering \\ $R V$ College of Engineering \\ Bengaluru, India
}

\begin{abstract}
Designing of a vehicle involves testing the vehicle under realistic simulations and scenarios to see how the vehicle responds. Manually performing all these tests would be expensive and there is also risk of human life. In this paper a vehicle driver model is modeled which can be used as a substitute to real drivers in simulations. The motion of the vehicle is considered in two components, longitudinal component and lateral component. Both these components are implemented using controllers. Model was built using MATLAB Simulink software and using path designer application, different paths were designed and the model was tested on them. The steering angle, velocity, acceleration, deceleration values generated for two test cases are displayed. The path followed by the driver model was also observed.
\end{abstract}

Keywords: Lateral Motion, Longitudinal Motion, MATLAB Simulink

\section{INTRODUCTION}

The social and economic losses due number of accidentst is quite significant. So a lot of work is done in testing the vehicle before it is sold for public use. There are two main types namely passive safety (design, seat-belts, air-bags etc) and active safety (camera and sensor information for predicting unforeseen situations. Therefore, testing is one of the most crucial step while designing any need component or technology. In automobile industry testing is done to understand how vehicle responds to different scenarios for e.g braking, recuperation, engine output whit respect to acceleration pedal position, steering angle to yaw motion produced etc. Tests are done also to check the functionality of different safety features in the vehicle. Driver model is essential to conduct such test. In this paper a control law based model is proposed to tracktrajectory, provided real-time steering, acceleration, brake commands on rapidly varyingoff-road terrain. Two controllers are used to implement the longitudinal and lateral motion. The longitudinal controller gives the acceleration and braking required and the lateral controller gives the steering angle required for the vehicle to stay in the designated path. Vehicle body 3DOF block is used as plant model for the controller model to work on. Reference generator block is used to provides the reference signal for thecontroller. Given the current position of the vehicle, it determines the reference position by searching for the closest point to the vehicle on the reference path. Reference generator also provides a reference velocity which is provided to the longitudinal controller block for it to maintain. The flow of the blocks implemented on simulink is provided in the flow diagram given below: 


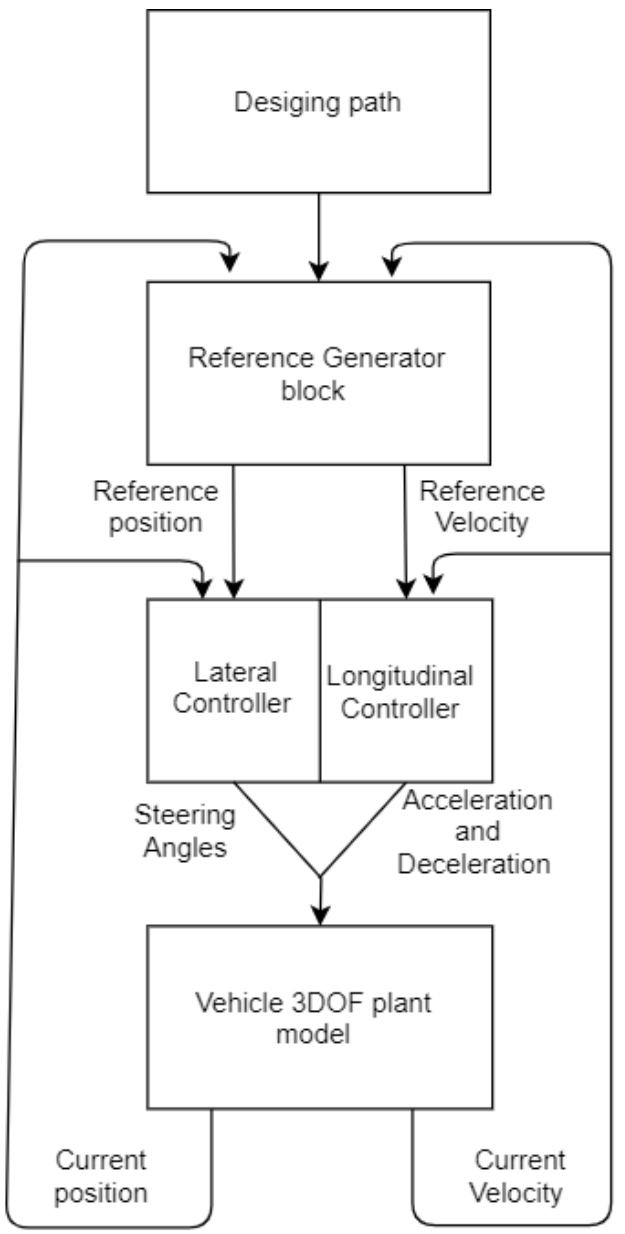

Figure 1. Vehicle Driver Model Flow Diagram

\section{DESIGNING PATH}

Creating new paths is essential to test the working of the model and find any boundary condition where the model might fail. Driving Scenario Designer application provided in simulink enables to design synthetic driving scenarios for testing the model. In the application a path is generated of required length and curvature. Ego Vehicle is added and is placed in the starting position. Generated path in the app can be used with the model by exporting the path as MATLAB code.



Figure 2. Path generated using DSD application 


\section{REFERENCE GENERATOR}

The lateral controller can the find the required steering angle based on the current position and the reference position it is required to move to. The reference finder block is a code written to find the next point on the reference path that the vehicle is required to move based on current position. With the current position returned by the vehicle plant model, the code runs through reference path points (which is imported from the path designer application) and calculates the distance between these points to current position. The point to which the distance is minimum is considered as reference point for this iteration. For next iterations the reference points after this is considered so that it doesn't repeat previous points. Velocity pro-filer block is used to generate reference velocity. It considers the curvature of the road and maximum acceleration and generates the reference velocity for the vehicle. The aim of this block is to avoid sudden jerks with increasing and decreasing velocity.

\section{LATERAL AND LONGITUDINAL CONTROLLER}

\subsection{Lateral Controller}

Lateral stanley controller is used as the lateral controller. To compute the steering angle command, the controller minimizes the positional error and the angle error of the current position with respect to the reference position. By determining a partial control law, given the kinematic equations of motion. Then, global asymptotic stability is proven, under the assumption of kinematic motion. Then the control law is modified to improve stability and tracking on the real system, by considering the dynamic equations of motion [1]. Considering the kinematic motion the steering law is defined as:

$$
\delta(t)= \begin{cases}\psi(t)+\arctan \frac{k e(t)}{v(t)} & \text { if }\left|\psi(t)+\arctan \frac{k e(t)}{v(t)}\right|<\delta_{\max } \\ \delta_{\max } & \text { if } \psi(t)+\arctan \frac{k e(t)}{v(t)} \geq \delta_{\max } \\ -\delta_{\max } & \text { if } \psi(t)+\arctan \frac{k e(t)}{v(t)} \leq-\delta_{\max }\end{cases}
$$

$\Psi(\mathrm{t})$ denotes the yaw angle and $\delta(\mathrm{t})$ angle of front wheel with respect to the vehicle.

\subsection{Longitudinal Controller}

The longitudinal controller generates acceleration and deceleration the vehicle should apply to match refernce velocity (generated by velocity pro-filer) from the current velocity. P.I (proportional-integral) controller is used which applies an accurate and responsive correction to a control function. The control signal, determines the value of acceleration and deceleration. The block saturates the acceleration and deceleration with respect to maximum and minimum acceleration parameter. The P.I control equation applied is:

$$
u(k)=\left(K_{\mathrm{p}}+K_{\mathrm{i}} \frac{T_{\mathrm{s}} z}{z-1}\right) e(k)
$$

\section{VEHICLE PLANT MODEL}

The Vehicle Body 3DOF block is used as plant model for the controller to consider as vehicle for simulation. This following block is provided by simulink and the parameters are changed with respect to requirement. The inputs for this block are steering angle and acceleration and deceleration values generated by lateral and longitudinal controller respectively. The model takes into consideration drag, mass and weight distribution between the axles due to the motion of the vehicle. In this bicycle model, the front wheel depict the front right and left wheels of the car, and the rear wheel depict the right rear wheel represents the rear right and left wheels of the car. The figure below represents the kinematics of the vehicle motion. 


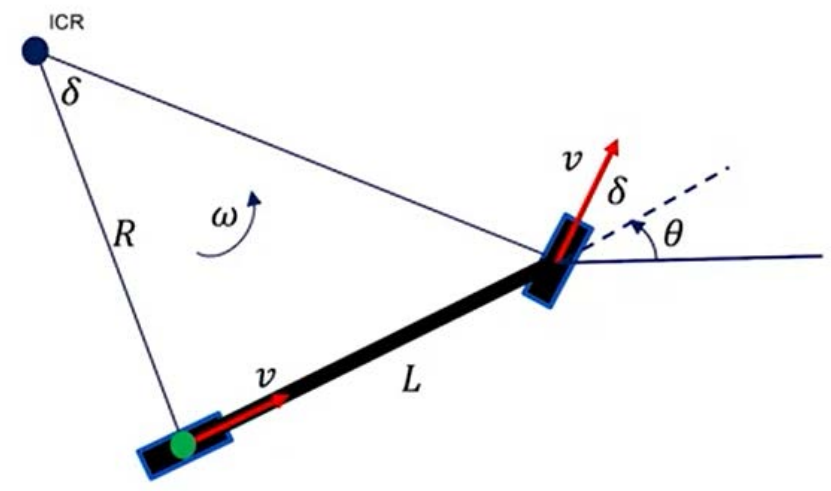

Figure 3. Kinematics of vehicle motion of bicycle model

Based on concepts of instantaneous center of rotation and similar triangle, the instantaneous velocity in $\mathrm{X}(\dot{\mathrm{x}})$ and $\mathrm{Y}(\dot{\mathrm{y}})$ direction and and the angle at which the vehicle is pointing is determined [2].

$$
\begin{aligned}
& \dot{x}_{r}=v \cos \theta \\
& \dot{y}_{r}=v \sin \theta \\
& \dot{\theta}=\frac{v \tan \delta}{L}
\end{aligned}
$$

\section{RESULTS}

The above developed model is tested with two different paths. The steering angles generatedfor each path by lateral controller is plotted. The steering limit is set to $35^{\circ}$. Lateral error generated by the steering controller is also plotted. Acceleration and deceleration values generated by longitudinal controller are plotted. Kp-2.5 and Ki-2.5 were chosen as P.I gain for longitudinal controller. The velocity obtained after inputting the steering, acceleration and deceleration to the vehicle plant model is plotted in the velocity vs time plot.

\subsection{S Shaped Path}

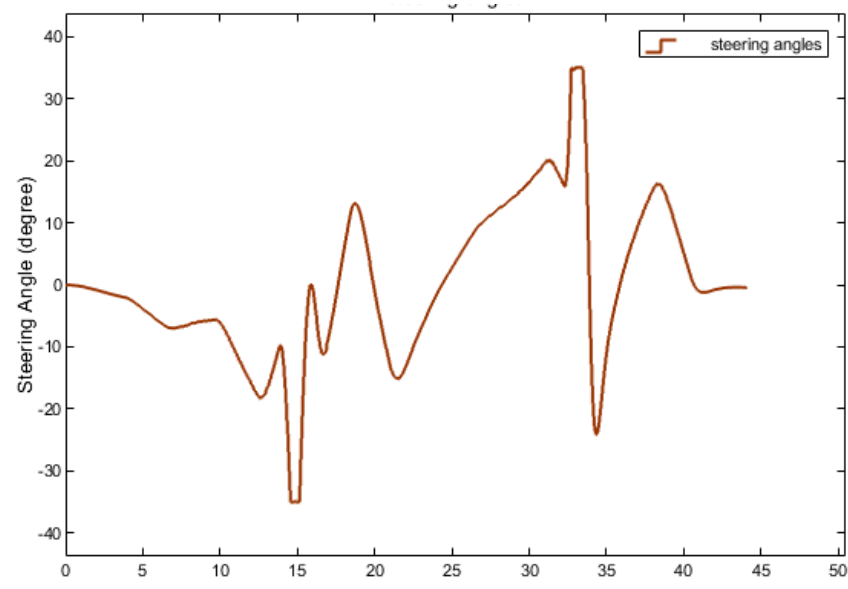

Figure 4. Steering Angles for $S$ path

The steering angles of the $\mathrm{S}$ path is plotted. The maximum steering limit is set to 35 degrees. When the vehicle approaches the curve the maximum values of steering is observed. 


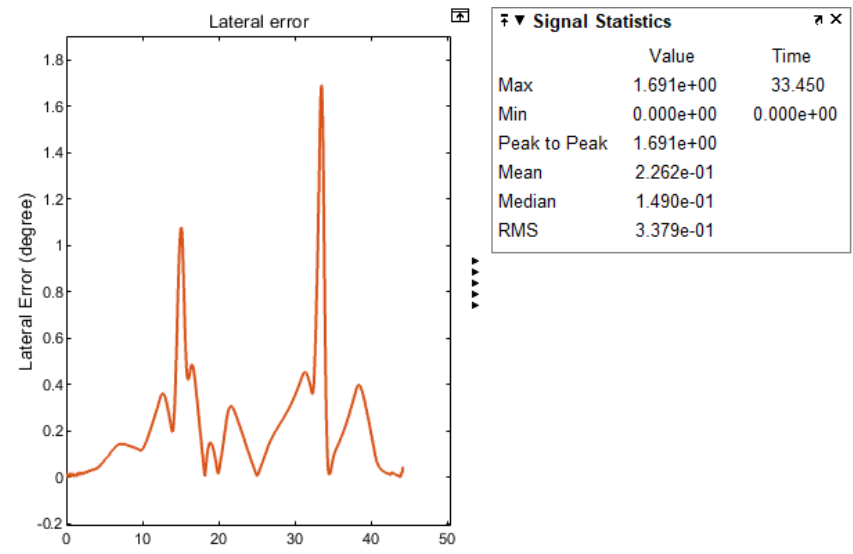

Figure 5. Lateral error observed for $\mathrm{S}$ path

The table in Fig.5. shows the average lateral error observed and other signal characteristics. The error calculation is done by comparing the reference path and the path generated due to steering angle generated from the diver model.

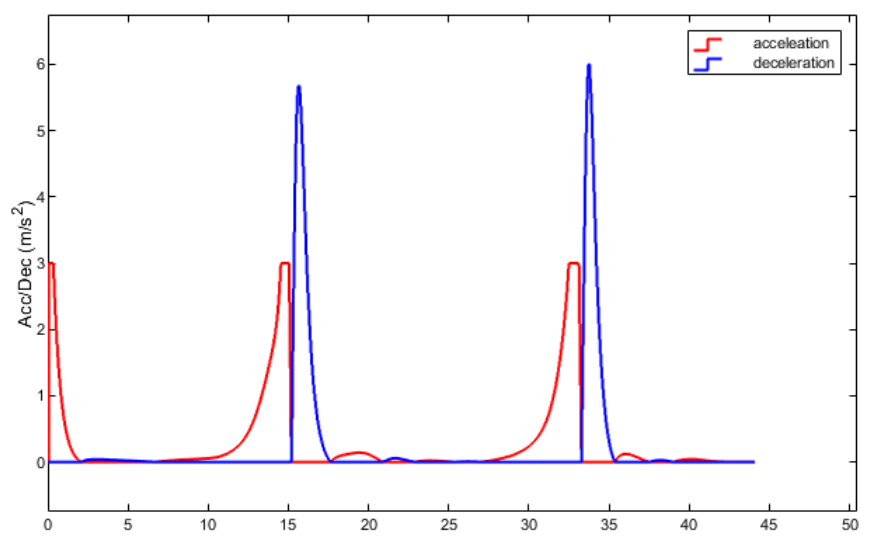

Figure 6. Acceleration and Deceleration plot for $\mathrm{S}$ path

The acceleration and deceleration for the $\mathrm{S}$ path are plotted. Initial acceleration is applied to reached a reference speed. This speed is maintained till the curves are approached where the brakes need to be applied which is symbolized by deceleration plot.

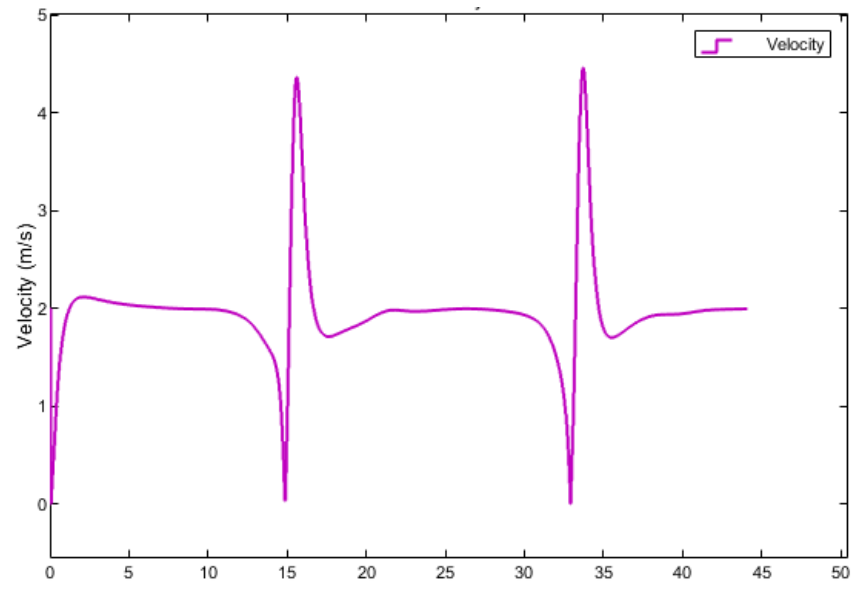

Figure 7. Velocity vs Time profile for $\mathrm{S}$ path

The vehicle's velocity vs time plot is shown. The vehicle tries to main a velocity around 14-16 kmph. When the curvature approaches the velocity decreases to allow the vehicle turn while remaining in the track limits. If the vehicle isn't slowed down, it will slip and slide away from the road. 


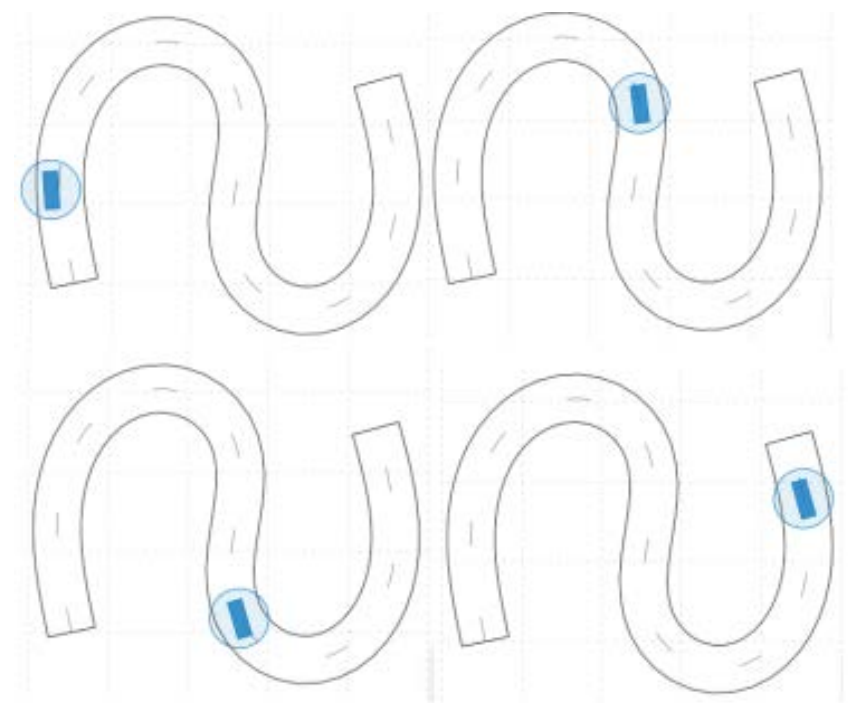

Figure 8. Path followed by the Vehicle on $\mathrm{S}$ path

The path followed by the vehicle is shown. The steering angles generated is input-ed to vehicle model which generated the points on the path. Using the bird-eye view on simulink the path generated can be viewed.

\subsection{O Shaped Path}

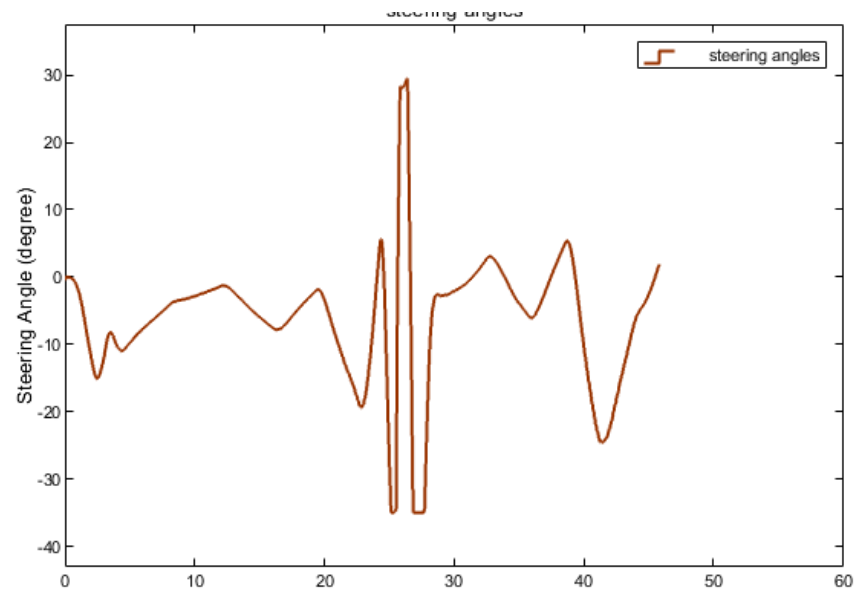

Figure 9. Steering Angles for $\mathrm{O}$ path

The steering angles of the $\mathrm{O}$ path is plotted. The maximum steering limit is set to 35 degrees. When the vehicle takes the U-turn, maximum variation in the steering angle is observed.

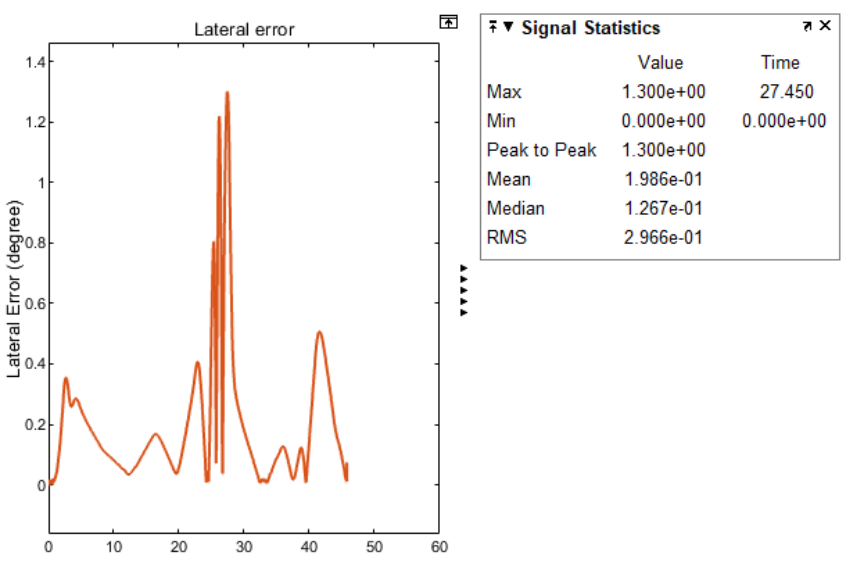

Figure 10. Lateral error observed for $O$ path

The table in Fig.5. shows the average lateral error observed and other signal characteristics. The error calculation is done by comparing the reference path and the path generated due to steering angle generated from the diver model. 


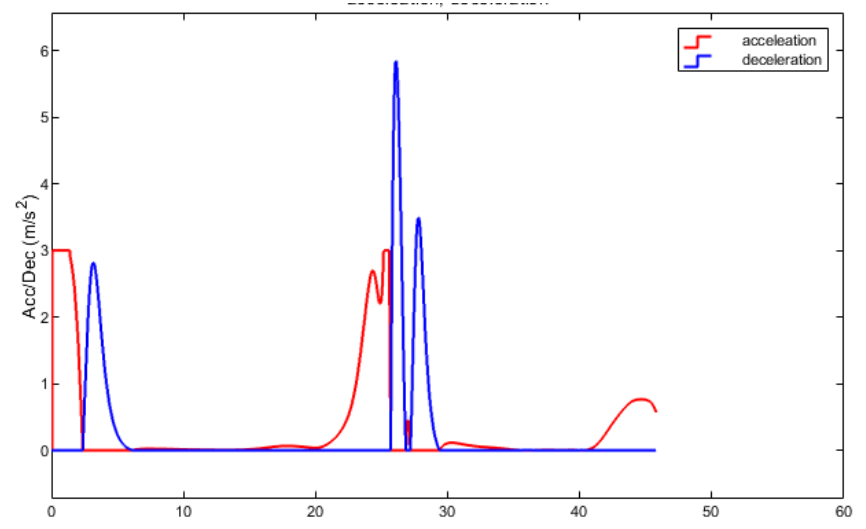

Figure 11. Acceleration and Deceleration plot for $O$ path

The acceleration and deceleration for the $\mathrm{O}$ path are plotted. Initial acceleration is applied to reached a reference speed. This speed is maintained till the curves are approached where the brakes need to be applied which is symbolized by deceleration plot. So the deceleration observed at the U-turn is justified.

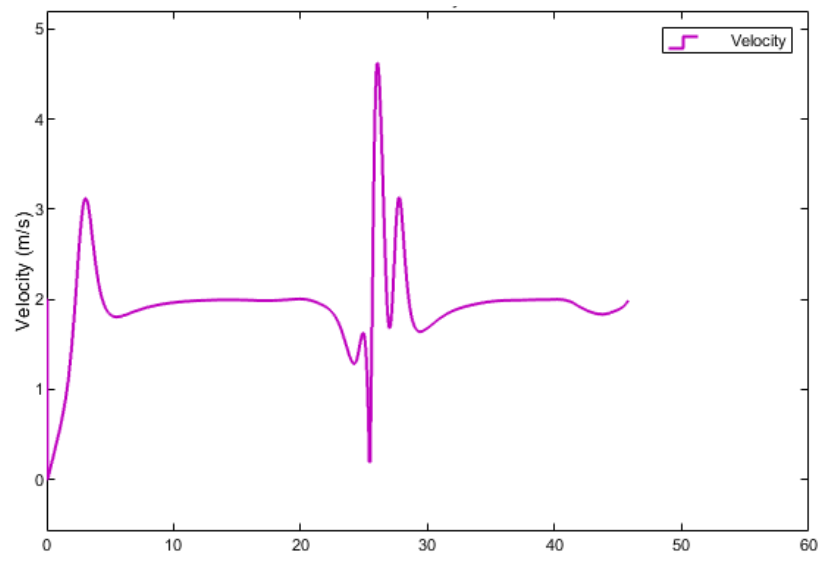

Figure 12. Velocity vs Time profile for $O$ path

The vehicle's velocity vs time plot is shown. The vehicle tries to main a velocity around 14-16 $\mathrm{kmph}$. When the U-turn approaches the velocity decreases to allow the vehicle turn while remaining in the track limits. If the vehicle isn't slowed down, it will slip and slide away from the road.



Figure 13. Path followed by the Vehicle on $O$ path

The path followed by the vehicle is shown. The steering angles generated is input-ed to vehicle model which generated the points on the path. Using the bird-eye view on simulink the path Vogenerates Isan be viewed. $_{2}$ 


\section{CONCLUSION}

Vehicle driver model which generates steering, acceleration and deceleration outputs for any given path is modeled and simulated. The birds-eye view of the path followed by the driver model is observed. This driver model can be used to run different simulation tests, which can act as substitute for real driver. One such test case where this model was to test and simulate powertrain simulation model which used the velocity-time profile generated by vehicle model. Currently the gain values for the controller is selected to have minimum error. For future scope, these gain values can be determined from real time test data so that the model depicts a human driver and can incorporate his different features.

\section{REFERENCES}

[1] G. M. Hoffmann, C. J. Tomlin, M. Montemerlo and S. Thrun, "Autonomous Automobile Trajectory Tracking for Off-Road Driving: Controller Design, Experimental Validation and Racing," 2007 American Control Conference, 2007, pp. 2296-2301, doi: 10.1109/ACC.2007.4282788.S. Wakitani et al..

[2] "Design of a Vehicle Driver Model Based on Database-Driven Control Approach," 2018 IEEE Conference on Control Technology and Applications (CCTA), 2018, pp. 840-845, doi: 10.1109/CCTA.2018.8511360.

[3] K. Oh and K. Yi, "A predictive driver model with physical constraints for closed loop simulation of vehicledriver system," 17th International IEEE Conference on Intelligent Transportation Systems (ITSC), 2014, pp. 3126-3131, doi: 10.1109/ITSC.2014.6958193.

[4] T. Levermore, A. Ordys and J. Deng, "A review of driver modelling," 2014 UKACC International Conference on Control (CONTROL), 2014, pp. 296-300, doi: 10.1109/CONTROL.2014.6915156.

[5] [5]Allen, R., Chrstos, J., Aponso, B., and Lee, D., "Driver/Vehicle Modeling and Simulation," SAE Technical Paper 2002-01-1568, 2002, doi: 10.4271/2002-01-1568

[6] Manfred Plöchl \& Johannes Edelmann (2007) Driver models in automobile dynamics application, Vehicle System Dynamics, 45:7-8, 699-741, DOI: 10.1080/00423110701432482

[7] A. Gray, Y. Gao, J. K. Hedrick and F. Borrelli, "Robust Predictive Control for semi-autonomous vehicles with an uncertain driver model," 2013 IEEE Intelligent Vehicles Symposium (IV), 2013, pp. 208-213, doi: 10.1109/IVS.2013.6629472.

[8] C. Miyajima et al., "Driver Modeling Based on Driving Behavior and Its Evaluation in Driver Identification," in Proceedings of the IEEE, vol. 95, no. 2, pp. 427-437, Feb. 2007, doi: 10.1109/JPROC.2006.888405.M. Young, The Techincal Writers Handbook. Mill Valley, CA: University Science, 1989.

[9] Gillespie, Thomas. Fundamentals of Vehicle Dynamics. Warrendale, PA: Society of Automotive Engineers (SAE), 1992.

[10] S. Schnelle, J. Wang, H. Su and R. Jagacinski, "A Personalizable Driver Steering Model Capable of Predicting Driver Behaviors in Vehicle Collision Avoidance Maneuvers," in IEEE Transactions on Human-Machine Systems, vol. 47, no. 5, pp. 625-635, Oct. 2017, doi: 10.1109/THMS.2016.2608930. 